\title{
Leptin, Resistin, and Proprotein Convertase Subtilisin/Kexin Type 9
}

\section{The Role of STAT3}

Chiara Macchi, ${ }^{*}$ Maria Francesca Greco, ${ }^{*}$ Margherita Botta, ${ }^{*}$ Paola Sperandeo, ${ }^{*}$ Paola Dongiovanni, ${ }^{\dagger}$ Luca Valenti,

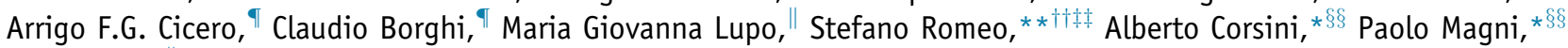
Nicola Ferri, " and Massimiliano Ruscica*

From the Departments of Pharmacological and Biomolecular Sciences $*$ and Pathophysiology and Transplantation, ${ }^{\ddagger}$ Università degli Studi di Milano, Milan, Italy; General Medicine and Metabolic Diseases ${ }^{\dagger}$ and Translational Medicine, Transfusion Medicine and Hematology, ${ }^{\S}$ Fondazione IRCCS Ca’ Granda Ospedale Maggiore Policlinico, Milan, Italy; the Department of Medicine and Surgery Sciences, ${ }^{\circledR}$ Alma Mater Studiorum University of Bologna, Bologna, Italy; the Department of Pharmaceutical and Pharmacological Sciences, "Università degli Studi di Padova, Padova, Italy; the Departments of Molecular and Clinical Medicine ${ }^{* *}$ and Cardiology, ${ }^{\ddagger \ddagger}$ Sahlgrenska Academy, University of Gothenburg, Gothenburg, Sweden; the Clinical Nutrition Unit, ${ }^{\dagger \dagger}$ Department of Medical and Surgical Science, Magna Graecia University, Catanzaro, Italy; and the IRCCS MultiMedica ${ }^{\S \S}$ Sesto S. Giovanni, Milan, Italy

Accepted for publication July $30,2020$.

Address correspondence to Massimiliano Ruscica, Ph.D., or Chiara Macchi, Ph.D., Department of Pharmacological and Biomolecular Sciences, Università degli Studi di Milano, Via Balzaretti 9, 20133, Milan, Italy. E-mail: chiara.macchi@unimi.itor massimiliano.ruscica@unimi.it.
In a condition of dysfunctional visceral fat depots, as in the case of obesity, alterations in adipokine levels may be detrimental for the cardiovascular system. The proinflammatory leptin and resistin adipokines have been described as possible links between obesity and atherosclerosis. The present study was aimed at evaluating whether proprotein convertase subtilisin/kexin type 9 (PCSK9), a key regulator of low-density lipoprotein metabolism, is induced by leptin and resistin through the involvement of the inflammatory pathway of STAT3. In HepG2 cells, leptin and resistin up-regulated PCSK9 gene and protein expression, as well as the phosphorylation of STAT3. Upon STAT3 silencing, leptin and resistin lost their ability to activate PCSK9. The knockdown of STAT3 did not affect the expression of leptin and resistin receptors or that of PCSK9. The analysis of the human PCSK9 promoter region showed that the two adipokines raised PCSK9 promoter activity via the involvement of a sterol regulatory element motif. In healthy males, a positive association between circulating leptin and PCSK9 levels was found only when the body mass index was $<25 \mathrm{~kg} / \mathrm{m}^{2}$. In conclusion, this study identified STAT3 as one of the molecular regulators of leptin- and resistin-mediated transcriptional induction of PCSK9. (Am J Pathol 2020, 190: 2226-2236; https://doi.org/10.1016/j.ajpath.2020.07.016)
Adipose tissue is an endocrine organ able to secrete active molecules, namely, adipokines, that contribute to regulating appetite and satiety, fat distribution, and energy expenditure. ${ }^{1}$ When the metabolic milieu is deranged, as in the case of obesity and adipose tissue metainflammation, adipokines may play a role in cardiovascular $(\mathrm{CV})$ disease, by promoting atherogenesis, plaque progression, and thrombosis. ${ }^{2}$ Roughly $80 \%$ of patients with coronary heart disease are overweight or obese. ${ }^{3}$ Among the huge variety of adipokines produced by adipose tissue, the proinflammatory adipokines leptin and resistin have been described as possible links between obesity and atherosclerosis. ${ }^{4,5}$
Supported by Cariplo Foundation grants 2015-0552 and 2018-0511 (M.R.), a MIUR Progetto Eccellenza grant (A.C.), Ricerca Finalizzata Ministero della Salute grant RF-2016-02364358 (L.V.), a Ricerca corrente Fondazione IRCCS Ca' Granda Ospedale Maggiore Policlinico grant (L.V.), and European Union (EU) Programme Horizon 2020 grant agreement 777377) for the project LITMUS "Liver Investigation: Testing Marker Utility in Steatohepatitis" (L.V.).

Disclosures: S.R. has been a consultant for, and received lecture fees and research grants from Amgen, Sanofi, and AstraZeneca; A.C. has received honoraria from AstraZeneca, Amgen, Sanofi, Recordati, Novartis, MSD, Mediolanum, DOC, Mylan, and Pfizer; L.V. has been a consultant for Gilead, Pfizer, AstraZeneca, Novo Nordisk, Intercept Pharmaceuticals, Diatech Pharmacogenetics, and IONIS. 
Leptin, a 16-kDa cytokine produced predominantly by adipose tissue, regulates feeding and promotes energy expenditure. This hormone is implicated also in the regulation of the immune system, autonomic, and $\mathrm{CV}$ regulation. ${ }^{6}$ Leptin exerts its biological actions by binding to a class I cytokine receptor encoded by $L E P R$ in humans. Among the six LepR isoforms (LepRa to LepRf), the LepRb is the only one with a full-length intracellular domain that allows the activation of a cell-signaling pathway. In response to leptin, Janus tyrosine kinase 2 (JAK2) phosphorylates LepR, triggering the recruitment of STAT3 through its SH2 domain. STAT3 is subsequently phosphorylated by JAK2, resulting in its dimerization and nuclear translocation in order to regulate the transcription of STAT3-target genes. ${ }^{7}$ Among the STAT proteins (STAT1, -2, -3, -4, -5a, -5b, and -6), STAT3 contributes to various metabolic processes such as hyperphagia and obesity, ${ }^{8}$ leptin being one of the major players in these events.

Resistin is a $12.5-\mathrm{kDa}$-sized C-terminal cysteine-rich signaling peptide secreted predominantly by adipose tissue and macrophages in humans and rodents. In humans, resistin adipose tissue expression and circulating levels are increased in overweight and obese subjects, strongly supporting the association with a raised $\mathrm{CV}$ risk in obese individuals. ${ }^{10}$ Among the designated receptors mediating resistin effects, for example, the inflammatory cascade, adenylyl cyclase associated protein 1 (CAP1) has been recently described as a bona fide resistin receptor. ${ }^{11}$

Proprotein convertase subtilisin/kexin type 9 (PCSK9), mainly secreted by the liver and to a lower extent by the intestine, post-translationally regulates the number of cellsurface low-density lipoprotein (LDL) receptors. At the transcriptional level, PCSK9 synthesis is largely controlled by the involvement of transcription factor families sterol regulatory element (SRE)-binding proteins (SREBPs) and by the acute-phase response transcriptional controller hepatocyte nuclear factor-1 (HNF-1) alpha. ${ }^{12}$ This last evidence and the knowledge that PCSK9 expression is also regulated by the proinflammatory cytokine tumor necrosis factor (TNF)- $\alpha$, in a suppressor of cytokine signaling (SOCS) 3-dependent manner, suggest a direct link between inflammation and the regulation of lipid metabolism via PCSK9. ${ }^{13}$

Considering that PCSK9 circulating levels have been associated with atherosclerosis and to support the hypothesis that the derangement in adipokine secretion is involved in obesity-associated CV risk, ${ }^{14}$ the aim of the present study was to evaluate whether leptin and resistin mediate PCSK9 activation through the inflammatory STAT3 pathway. Indeed, the JAK/STAT signaling pathway, a pillar downstream mediator of a variety of cytokines, is dysregulated in metabolic diseases including obesity. ${ }^{15}$

\section{Materials and Methods}

\section{Cell Cultures}

The human hepatocellular carcinoma cell line, HepG2, was routinely cultured in 10\% fetal bovine serum (FBS)/Dulbecco's modified Eagle's medium (DMEM) supplemented with penicillin, streptomycin, nonessential amino acids, and sodium pyruvate (all from Sigma-Aldrich, Milan, Italy). For the experiments, cells were starved overnight and then incubated with DMEM containing 10\% human lipoprotein plasma deprived serum (LPDS).

\section{Reagents and Antibodies}

DMEM, trypsin EDTA, penicillin, streptomycin, nonessential amino acid solution, FBS, disposable culture flasks, and petri dishes were from Merck (Milan, Italy). Molecular weight protein standard, precast polyacrylamide gels ( $4 \%$ to $12 \%$ ), bicinchoninic acid assay for determination of protein concentrations, Maxima First Strand cDNA, and Maxima SYBR Green/Fluorescein qPCR Master Mix were all from Life Technologies (Monza, Italy). Bovine Serum Albumin was from Sigma-Aldrich. Human recombinant leptin (Sigma-Aldrich) and resistin (BioVision, Milpitas, CA) were used at $100 \mathrm{ng} / \mathrm{mL}$. Human recombinant PCSK9 was used at $2.5 \mu \mathrm{g} / \mathrm{mL}$ (item 20631; Cayman Chemicals, Ann Arbor, MI). ${ }^{16}$

\section{Transfection of siRNA}

ON-TARGET plus SMART pool siRNA directed to STAT3 and PCSK9 or scramble control were from Dharmacon (Carlo Erba Reagents, Milan, Italy). Transfections were performed by using siLentFect Lipid Reagent (Bio-Rad Laboratories, Hercules, CA) according to the manufacturer's protocol. The day before the transfection, HepG2 cells were seeded in DMEM/10\% FBS at a density of $6 \times 10^{5} /$ well (6-well tray). Cells were then transfected with $40 \mathrm{nmol} / \mathrm{L}$ siRNA. Forty-eight hours after the transfection, the medium was replaced with DMEM/10\% LPDS \pm leptin (100 $\mathrm{ng} / \mathrm{mL}$ ) or resistin $(100 \mathrm{ng} / \mathrm{mL})$ for an additional 24 hours before performing quantitative PCR analysis.

\section{In Silico Analyses for STAT3 Binding Motifs on Human PCSK9 Promoter}

Human, gorilla, mouse, and rat PCSK9 promoter sequences (up to 2000 bp upstream from the start codon) were retrieved from Ensembl Genome Browser ${ }^{17}$ by using the latest released genome assembly (GRCh38.p13, Kamilah_GGO_v0, GRCm38.p6, and Rnor_6.0 for human, gorilla, mouse, and rat, respectively). JASPAR (http://jaspar.genereg.net, last accessed July 2020), an open-access database of curated, nonredundant transcription factor binding profiles modelled as position-specific weight matrices, was used to scan the selected PCSK9 promoter region for any STAT3 binding site. ${ }^{18}$ The relative profile score threshold-defined as the minimum 
relative score required for reporting a match between the transcription factor binding model and the imputed sequence-was set at $80 \%$. The FIMO tool, from the open-access MEME Suite 5.1.1 (http://meme-suite.org, last accessed July 2020), was used to double-check the results obtained from JASPAR. ${ }^{19,20}$ The STAT3 position-specific weight matrix profile was retrieved from JASPAR and imputed in FIMO, and the selected PCSK9 promoter region was scanned. Matches with $P \leq 0.0001$ were taken into account. The same analyses were performed for SRE and HNF-1.

\section{Generation of PCSK9 Promoter Luciferase Reporters with a STAT3 Binding Site Deletion}

D1-STAT3-mut plasmid was generated by deleting the STAT3 binding sequence predicted by JASPER in the $P C S K 9$ promoter sequence in plasmid D1. Plasmid D1 is a derivative of the commercial promoter-reporter vector pGL3-Basic, generated by cloning the human PCSK9 promoter fragment spanning - 1711 to -94 upstream of the $l u c$ reporter gene. Deletion of the STAT3 sequence (CTTCTGGAAAG), spanning -916 to -906 in the PCSK 9 promoter, was obtained by two-step PCR according to the following protocol. Two fragments flanking the putative STAT3 sequence were PCR amplified using D1 plasmid as a template and the following primer pairs: AP679 (5'-CGACGAGGTACCGAGCTCGGATCCACTAGTAAC- $3^{\prime}$ ) and AP680 (5'-ATTCAATTTGCAAAGATTC-3') and generate the upstream fragment, and AP681 (5'-CAAATTGAATCTGAGCTTGTGCCTACCATAG-3') and AP682 (5'CCCAAGCTTACTGTGCAGGAGCTGAAGTTC- $3^{\prime}$ ) to generate the downstream fragment. Primer AP679 and AP682 sequences contain the KpnI and HindIII restriction sites, respectively. The upstream and downstream fragments were used as templates for a second round of PCR amplification using AP679 and AP682 primers. The final PCR product was cloned in the D1 plasmid between KpnI and HindIII restriction sites in place of the PCSK 9 promoter region, generating the D1-STAT3 plasmid. The deletion of the STAT3 region from the PCSK9 promoter in the D1-STAT3 plasmid was confirmed by DNA sequencing.

\section{Transfections of Reporter Constructs}

HepG2 cells were transfected with the plasmid PCSK9 pGL3-PCSK9-D4 containing the $5^{\prime}$ flanking region of the
PCSK 9 gene from -440 to -94 , relative to the ATG start codon in front of the luciferase coding sequence. The promoter constructs contain wild-type, SRE mutated (SRE-mu), and HNF-1 alpha mutated (HNF1-mu) sequences. ${ }^{21}$ To measure the human PCSK9 promoter activity, HepG2 cells were seeded in 48 -well plates at a density of $4 \times 10^{5}$ cells per well. The day after, cells were transiently transfected with pGL3-PCSK9-D1 (wild-type and STAT3 mutated) and with pGL3-PCSK9-D4 plasmids (wild-type, SRE-mu, and HNF1-mu), with TurboFect transfection reagent (Thermo Fisher Scientific, Waltham, MA). Forty-eight hours after the transfection, cells were incubated with DMEM/10\% LPDS \pm leptin $(100 \mathrm{ng} / \mathrm{mL})$, resistin $(100 \mathrm{ng} / \mathrm{mL})$, or simvastatin $(20 \mu \mathrm{mol} / \mathrm{L})$ for an additional 24 hours. Luciferase activity was measured by using Neolite reagent (Perkin Elmer, Milan, Italy) according to the manufacturer's instructions.

\section{RNA Preparation and Quantitative Real-Time PCR}

Total RNA was extracted with the iScript Sample Preparation Buffer cDNA synthesis preparation reagents (Bio-Rad Laboratories) according to manufacturer's instructions or by spin column (Qiagen, Milan, Italy). Reverse transcriptionpolymerase first-strand cDNA synthesis was performed by using Maxima First Strand cDNA synthesis kit (Thermo Fisher Scientific). Quantitative PCR was then performed by using the Thermo SYBR Green/ROX qPCR Master Mix kit (Thermo Fisher Scientific) and specific primers for selected genes. The analyses were performed with the 9600 Bio-Rad Real-Time PCR Detection Systems (Bio-Rad Laboratories). The primer sequences are listed in Table 1. PCR cycling conditions were as follows: $94^{\circ} \mathrm{C}$ for 5 minutes, 40 cycles at $94^{\circ} \mathrm{C}$ for 15 seconds, and $60^{\circ} \mathrm{C}$ for 30 seconds. Data are expressed as $\mathrm{Ct}$ values and used for the relative quantification of targets with the $2^{-\Delta \Delta C t}$ calculation.

\section{Western Blot Analysis}

Total cytosolic protein extracts of HepG2 were obtained by collecting cells in $70 \mu \mathrm{L}$ of Mammalian Protein Extraction Reagents (Thermo Fisher Scientific) containing a cocktail of protease and phosphatase inhibitors (Roche Diagnostics, Basel, Switzerland). Ten micrograms of proteins and a

Table 1 List and Sequence of Primers Used in This Study

\begin{tabular}{lll}
\hline Primers & Forward & Reverse \\
\hline PCSK9 & 5'-CCTGCGCGTGTCAACT-3' & $5^{\prime}$-GCTGGCTTTTCCGAAACTC-3' \\
STAT3 & 5'-CAGCAGCTTGACACACGGTA-3' & $5^{\prime}$-AAACACCAAAGTGGCATGT-3' \\
ACTB & 5'-TTCTACAATGAGCTGCGTGTG-3' & $5^{\prime}-$ GGGGTGTTGAAGGTCTCAAA-3' \\
LEPR & 5'-TACTTTGGAAGCCCCTGATG-3' & $5^{\prime}-$ AAGCACTGAGTGACTGCACG-3' \\
CAP1 & 5'-ACTGGCCTGGAGCAAAACG-3' & $5^{\prime}-$ CGGCAGAGGGTCCAGATG-3' \\
IL6 & 5'-ACCCCCAGGAGAAGATTCCA-3' & $5^{\prime}$-GGTTGTTTTCTGCCAGTGCC-3' \\
\hline
\end{tabular}

ACTB, $\beta$-actin; CAP1, adenylyl cyclase associated protein 1; LEPR, leptin receptor; PCSK9, proprotein convertase subtilisin/kexin type 9. 
A

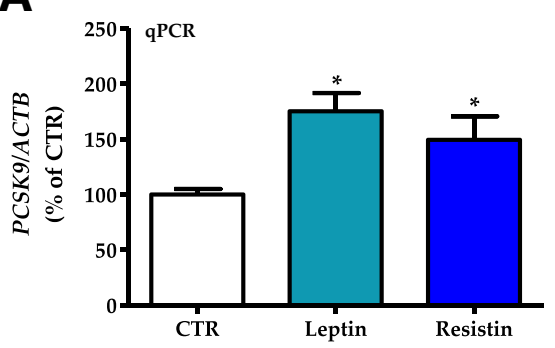

D

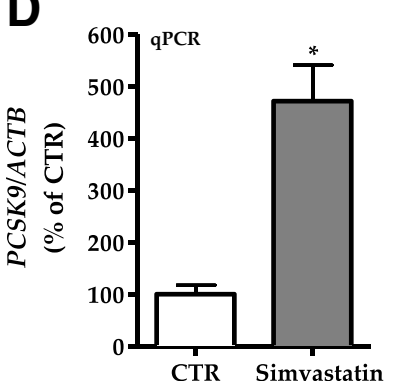

B

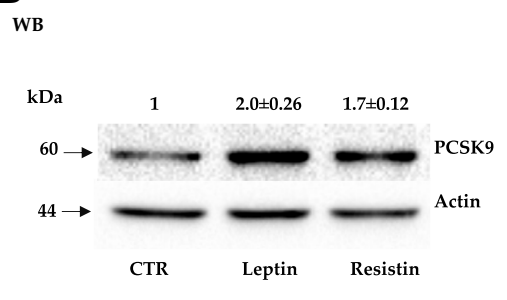

E

WB

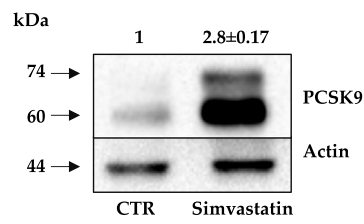

C

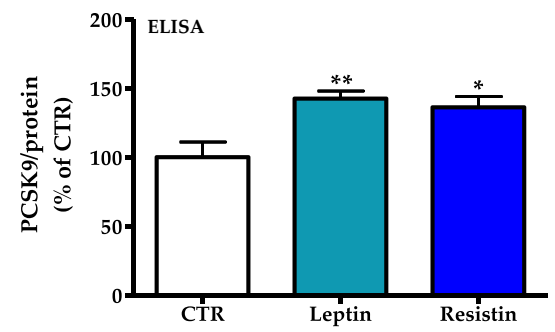

$\mathbf{F}$

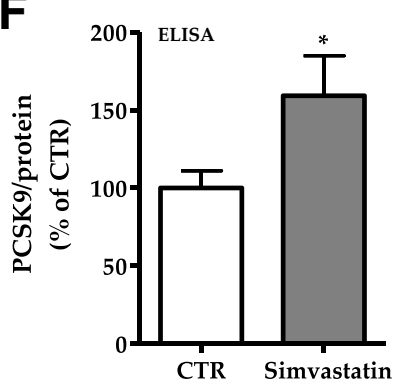

Figure 1 HepG2 cells were seeded in DMEM/10\% fetal bovine serum. Cells were incubated with leptin (100 ng/mL) or resistin (100 ng/mL) in DMEM supplemented with $10 \%$ LPDS for 24 hours. Simvastatin $(20 \mu \mathrm{mol} / \mathrm{L})$ has been used as a positive control. At the end of the incubation, total RNA and proteins were extracted. $\beta$-Actin was used as housekeeping for both gene (ACTB) and protein expression. A and D: PCSK9 gene expression (qPCR). B and E: PCSK9 protein expression (WB). C and F: PCSK9 levels assayed by ELISA (data were normalized for the total amount of proteins). The values above the blots represent the densitometric readings. $n \geq 3$ independent experiments $(\mathbf{A}-\mathbf{F})$. Differences between groups have been assessed by $t$-test $(\mathbf{D}$ and $\mathbf{F})$ or one-way analysis of variance $(\mathbf{A}$ and $\mathbf{C}) .{ }^{*} P<0.05,{ }^{*} P<0.01$ versus control. ACTB, $\beta$-actin gene; CTR, control (medium alone); DMEM, Dulbecco's modified Eagle's medium; ELISA, enzyme-linked immunosorbent assay; LPDS, human lipoprotein plasma deprived serum; PCSK9, Proprotein convertase subtilisin/kexin type 9; qPCR, quantitative real-time PCR; WB, Western blot.

molecular mass marker (Novex Sharp Protein Standard, Invitrogen; Thermo Fisher Scientific) were separated on $4 \%$ to $12 \%$ SDS-PAGE (Novex NuPAGE $4 \%$ to $12 \%$ Bis-Tris MiniGels; Invitrogen; Thermo Fisher Scientific) under denaturing and reducing conditions. Proteins were then transferred to a nitrocellulose membrane by using the iBlotTM Gel Transfer Device (Invitrogen; Thermo Fisher Scientific). The membranes were washed with Tris-buffered saline-Tween 20, and nonspecific binding sites were blocked in Tris-buffered saline-Tween 20 containing 5\% bovine serum albumin (Sigma-Aldrich) for 90 minutes at room temperature. The blots were incubated overnight at $4^{\circ} \mathrm{C}$ with a diluted solution ( $5 \%$ bovine serum albumin or nonfat dry milk) of the human primary antibodies (listed below). Membranes were washed with Tris-buffered saline-Tween 2 and then exposed for 90 minutes at room temperature to a diluted solution (5\% nonfat dry milk) of the secondary antibodies (anti-mouse and anti-rabbit peroxidase-conjugated secondary antibodies; New England Biolabs, Ipswich, MA). Immunoreactive bands were detected by exposing the membranes to Clarity Western ECL chemiluminescent substrates (Bio-Rad Laboratories) for 5 minutes, and images were acquired with a ChemiDoc XRS System (Bio-Rad Laboratories). Densitometric readings were evaluated using the ImageLab software version 6.0.1 (Bio-Rad Laboratories). The dilution of the human primary antibodies were: PCSK9 (1:1000; Genetex, Irvine, CA), STAT3 (1:1000; Cell Signaling Technology, Danvers, MA), pSTAT3 (1:10,000;
Abcam, Cambridge, UK), actin (1:1000; Santa Cruz Biotechnology, Santa Cruz, CA), tubulin (1:2000; SigmaAldrich), and vinculin (1:1000; Genetex).

\section{Study Population}

The association between PCSK9, and leptin and resistin plasma levels was evaluated in 149 healthy male subjects from the Brisighella Heart Study. This study is a longitudinal population study on a randomized sample representative of the entire population of Brisighella, a rural Northern Italian village. The study has been active since 1972 and has been performed in agreement with the Declaration of Helsinki. The protocol was approved by the institutional ethics board of the University Hospital of Bologna. ${ }^{22}$

\section{ELISA Assays}

Plasma PCSK9 concentrations were measured by a commercial enzyme-linked immunosorbent assay (ELISA) kit (R\&D Systems, Minneapolis, MN). The minimum detectable PCSK9 concentration was $0.219 \mathrm{ng} / \mathrm{mL} .^{23}$ Concerning the evaluation of PCSK9 in the conditioned medium, HepG2 cells were cultured in 12-well plates $\left(6 \times 10^{5}\right.$ cells/well). The medium $(200 \mu \mathrm{L})$ was collected after 24-hour treatment with leptin, resistin, and simvastatin. The samples were not 
A
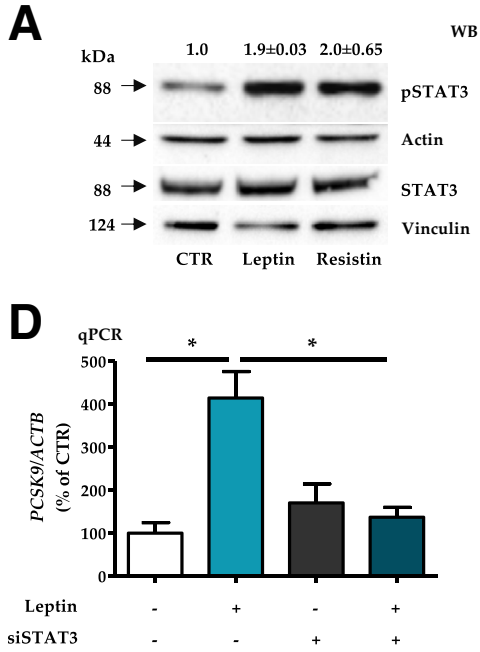

B
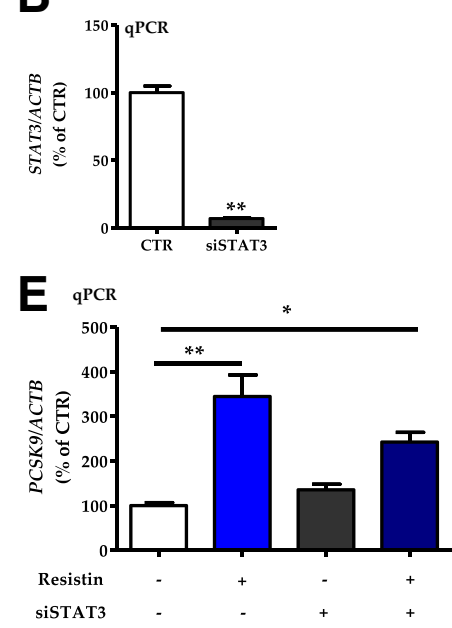

C

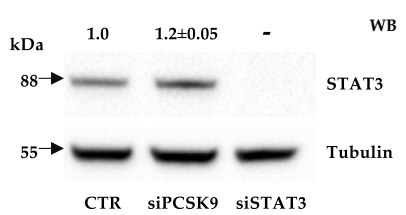

$\mathbf{F}$

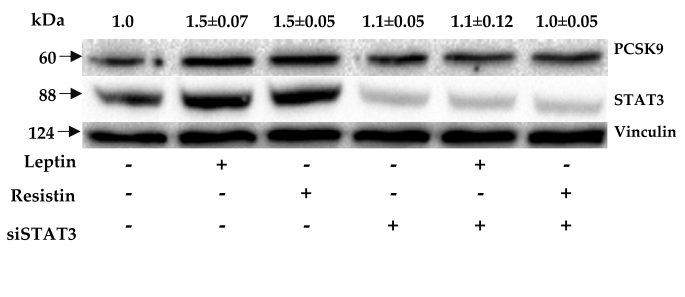

Figure 2 A: HepG2 cells were seeded in DMEM/10\% FBS. After 48 hours, the medium was replaced with DMEM with $10 \%$ LPDS supplemented with leptin (100 $\mathrm{ng} / \mathrm{mL}$ ) and resistin $(100 \mathrm{ng} / \mathrm{mL})$. After 24 hours, the expression of PSTAT3 ${ }^{\mathrm{Y} 705}$ and STAT3 was evaluated. $\beta$-Actin and vinculin were used as housekeeping proteins. B and C: HepG2 cells were seeded in DMEM/10\% FBS and the day after transfected with siRNA scramble and siRNA anti-STAT3 or siRNA anti-PCSK9. After 48 hours, the expression of STAT3 was evaluated. To normalize the data, ACTB was used as housekeeping gene and tubulin as housekeeping protein. D and E: HepG2 transfected with siRNA scramble and siRNA anti-STAT3 were or were not treated with leptin $(100 \mathrm{ng} / \mathrm{mL})$ and resistin $(100 \mathrm{ng} / \mathrm{mL})$, and the gene expression of PCSK9 was evaluated by qPCR. Actin was used as a housekeeping gene. F: HepG2 transfected with siRNA scramble and siRNA anti-STAT3 were or were not treated with leptin $(100 \mathrm{ng} / \mathrm{mL})$ and resistin $(100 \mathrm{ng} / \mathrm{mL})$, and the protein expression of PCSK9 was evaluated by WB. Vinculin was used as a housekeeping protein to normalize the data. In $\mathbf{A}, \mathbf{C}$, and $\mathbf{F}$, the values above the blots represent the densitometric readings evaluated by using ImageLab software version 6.0 .1 . At least three independent experiments were conducted. Differences between groups were assessed by $t$-test $(\mathbf{B})$ and one-way analysis of variance $(\mathbf{D}$ and $\mathbf{E})$. ${ }^{*} P<0.05$, $* * P<0.01$. ACTB, $\beta$-Actin; CTR, control (medium alone); DMEM, Dulbecco's modified Eagle's medium; FBS, fetal bovine serum; LPDS, human lipoprotein plasma deprived serum; PCSK9, proprotein convertase subtilisin/kexin type 9; qPCR, quantitative real-time PCR; siRNA, silencing RNA; WB, Western blot.

diluted, and results were normalized for the total amount of proteins.

Plasma leptin concentrations were measured by a commercial ELISA kit (R\&D Systems). The minimum detectable dose of human leptin is typically $<7.8 \mathrm{pg} / \mathrm{mL}^{24}$

\section{Analysis of the Data}

Statistical analysis was performed using the Prism statistical analysis package version 6.0 (GraphPad Software, San Diego, CA). Data are given as means \pm SD of three independent experiments. When possible, $P$ values were determined by $t$-test. Otherwise, differences between treatment groups were evaluated by one-way analysis of variance. A probability value of $P<0.05$ was considered statistically significant.

\section{Results}

\section{Leptin and Resistin Induce PCSK9}

First, a time course experiment $(4,8,16$, and 24 hours) was conducted to evaluate the gene expression of PCSK 9 upon leptin $(100 \mathrm{ng} / \mathrm{mL})$ and resistin $(100 \mathrm{ng} / \mathrm{mL})$ treatment (Supplemental Figure S1). Incubation of HepG2 cells with human recombinant leptin and resistin resulted in a significant rise in PCSK9 gene expression of roughly $+50 \%$ (Figure 1A) after 24 hours. Compared with medium alone, protein expression, evaluated by Western blot analysis, was increased roughly by 2- and 1.7-fold, upon treatment with leptin and resistin, respectively (Figure 1B). Simvastatin (20 $\mu \mathrm{mol} / \mathrm{L})$ - used as a positive control-raised both PCSK9 gene and protein expression (Figure 1, D and E). To corroborate Western blot analyses, the release of PCSK9 was measured. In the HepG2-conditioned medium, the levels of PCSK9 were increased by $42.6 \%(P<0.01)$ and $36.3 \%(P<0.05)$ following leptin and resistin treatments, respectively (Figure $1 \mathrm{C})$. Simvastatin $(20 \mu \mathrm{mol} / \mathrm{L})$ was used as a positive control (Figure $1 \mathrm{~F}$ ).

\section{STAT3 Mediates Leptin- and Resistin-Driven PCSK9 Activation}

In this cell-based model, 24-hour treatment with leptin and resistin phosphorylated STAT3 (pSTAT3 ${ }^{\mathrm{Y} 705}$ ) without affecting the protein expression of total STAT3 (Figure 2A). To follow up the hypothesis that STAT3 was involved in leptin- and resistin-driven PCSK9 activation, HepG2 cells were knocked down for STAT3. Transfection with specific siRNA anti-STAT3 fully abolished the gene and protein expression of STAT3 (Figure 2, B and C) without affecting that of PCSK9 (Figure 2, D-F). Under these experimental conditions, leptin and resistin did not up-regulate PCSK9 expression (gene and protein). These data suggest that PCSK9 up-regulation by leptin and resistin in hepatocytes requires STAT3. To exclude any effect of PCSK9 on STAT3 expression, it was shown that silencing PCSK9 did not result in any change in STAT3 levels (Figure 2C). 
Table 2 In Silico Search of STAT3 Binding Sites on the Proximal PCSK9 Promoter Region

\begin{tabular}{lcclll}
\hline Predicted site sequence & Start & End & Strand & JASPAR score, \% & FIM0 threshold, $P$ value \\
\hline CTTCTGGAAAG & -916 & -906 & + & 95.92 & $2.07 \times 10^{-5}$ \\
CTTCCAGAAAG & -1082 & -1072 & + & 93.06 & $7.44 \times 10^{-4}$ \\
CTTTCTGGAAG & -1072 & -1082 & - & 92.42 & $1.01 \times 10^{-4}$ \\
ATACTGGGAAG & -1091 & -1101 & - & 89.88 & $2.93 \times 10^{-4}$ \\
TTGCCTGTAAT & -1385 & -1395 & - & 88.45 & $4.46 \times 10^{-4}$ \\
CAGCAGGGAAA & -1485 & -1475 & + & 87.79 & $4.61 \times 10^{-4}$ \\
GTTCAAGAAAT & -950 & -960 & - & 87.27 & $7.13 \times 10^{-4}$ \\
GTGAAGGGAAA & -2184 & -2174 & + & 87.08 & $6.22 \times 10^{-4}$ \\
\hline
\end{tabular}

PCSK9, proprotein convertase subtilisin kexin type 9.

Moreover, application of siSTAT3 did not change the gene expression of leptin $(L E P R)$ and resistin $(C A P 1)$ receptors (Supplemental Figure S2).

\section{STAT3 and PCSK9 Transcriptional Activity}

The involvement of STAT3 in the PCSK9 pathway was further investigated at the transcriptional level. First, an in silico search of STAT3 binding sites on proximal PCSK9 promoter region was performed by using JASPAR database and FIMO tool from MEME Suite 5.1.1. The analyses revealed a strong
STAT3 binding site (JASPAR score: 96\%; FIMO threshold: $P=2.07 \times 10^{-5}$ ) spanning the region -916 to -906 in the human PCSK9 promoter (Table 2). This region is upstream from the Sp1/HNF1- $\alpha / \mathrm{SRE}$ triad already published by $\mathrm{Li}$ et $\mathrm{al}^{21}$ (Figure 3A). To corroborate this finding, a similar analysis was performed on gorilla, mouse, and rat PCSK 9 promoters: a multiple alignment of the four sequences was generated thanks to Nucleotide BLAST Alignment tool by NCBI (https://blast.ncbi.nlm.nih.gov/Blast.cgi) (Figure 3A). The mild degeneration in the STAT3 consensus sequence has been already reported in the literature. ${ }^{25}$ After transfection with
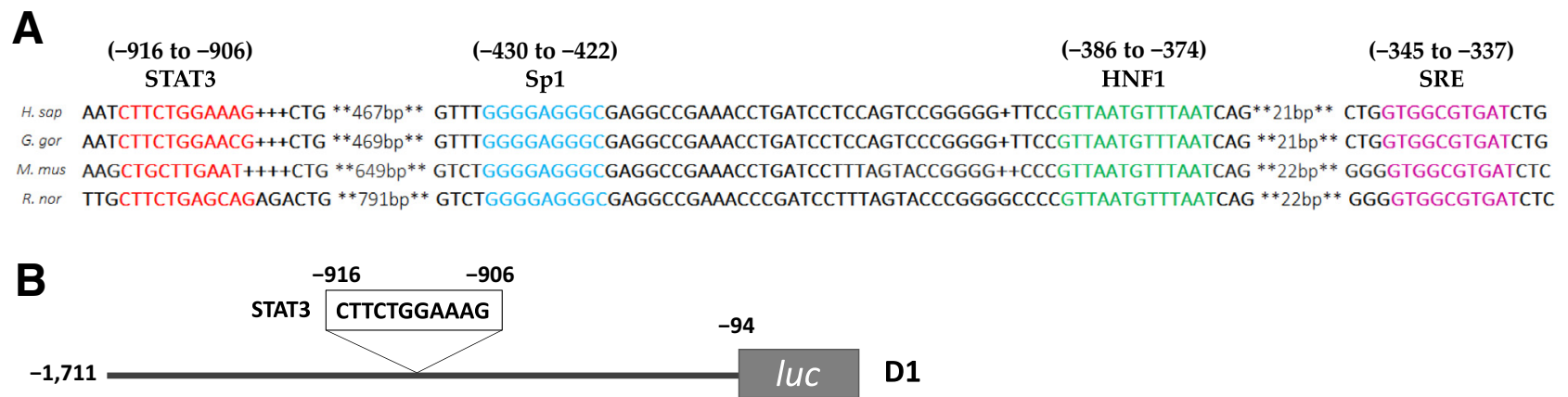

C

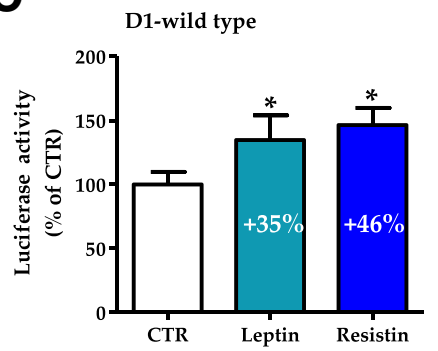

D1-STAT3-mu

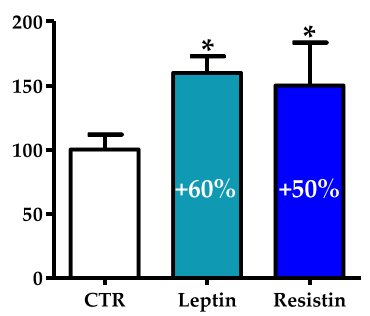

D

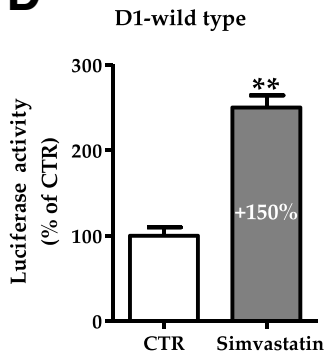

D1-STAT3-mu

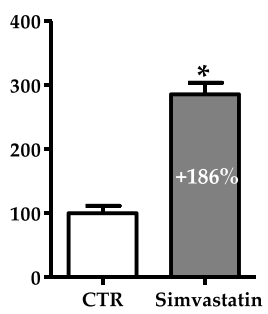

Figure 3 A: Sequence comparison of proximal regions of PCSK9 promoter of human, gorilla, mouse, and rat species. B: Schematic representation of the human PCSK9 promoter-luciferase reporter plasmid D1. Positions refer to the nucleotide preceding the ATG start codon of the human PCSK9 gene, arbitrarily designated as -1. Positions -94 and -1711 indicate the $3^{\prime}$ and the $5^{\prime}$ ends of the PCSK9 promoter insert, respectively. Positions of the $5^{\prime}$ and $3^{\prime}$ ends of the STAT3 binding sequence are indicated. C and D: HepG2 cells were transfected with pGL3-PCSK9-D1 and pGL3-PCSK9-D1-STAT3-mutated. The day after the transfection, the medium was replaced with Dulbecco's modified Eagle's medium containing 10\% LPDS supplemented with leptin (100 ng/mL), resistin (100 ng/mL), or simvastatin (20 $\mu$ mol/L). After an additional 24 hours, luciferase activities were determined by Neolite reagent. Differences between groups were assessed by $t$-test (D) or one-way analysis of variance (C). Numbers inside the bars represent the percentage of increment. Nucleotides in red refer to the STAT3 sequence in the PCSK9 promoter region; nucleotides in blue refer to the Sp1 sequence in the PCSK9 promoter region; nucleotides in green refer to the HNF-1 sequence in the PCSK9 promoter region; nucleotides in purple refer to the SRE sequence in the PCSK9 promoter region (A); double asterisks in A refer to bp spanning between the interregion sequences of the PCSK9 promoter region. $n \geq 3$ independent experiments $(\mathbf{C}$ and $\mathbf{D}) .{ }^{*} P<0.05,{ }^{* *} P<0.01$ versus control. CTR, control (medium alone); HNF1, hepatocyte nuclear factor-1; LPDS, human lipoprotein plasma deprived serum; PCSK9, proprotein convertase subtilisin/kexin type 9; Sp1, proximal specificity protein 1; SRE, sterol regulatory element. 

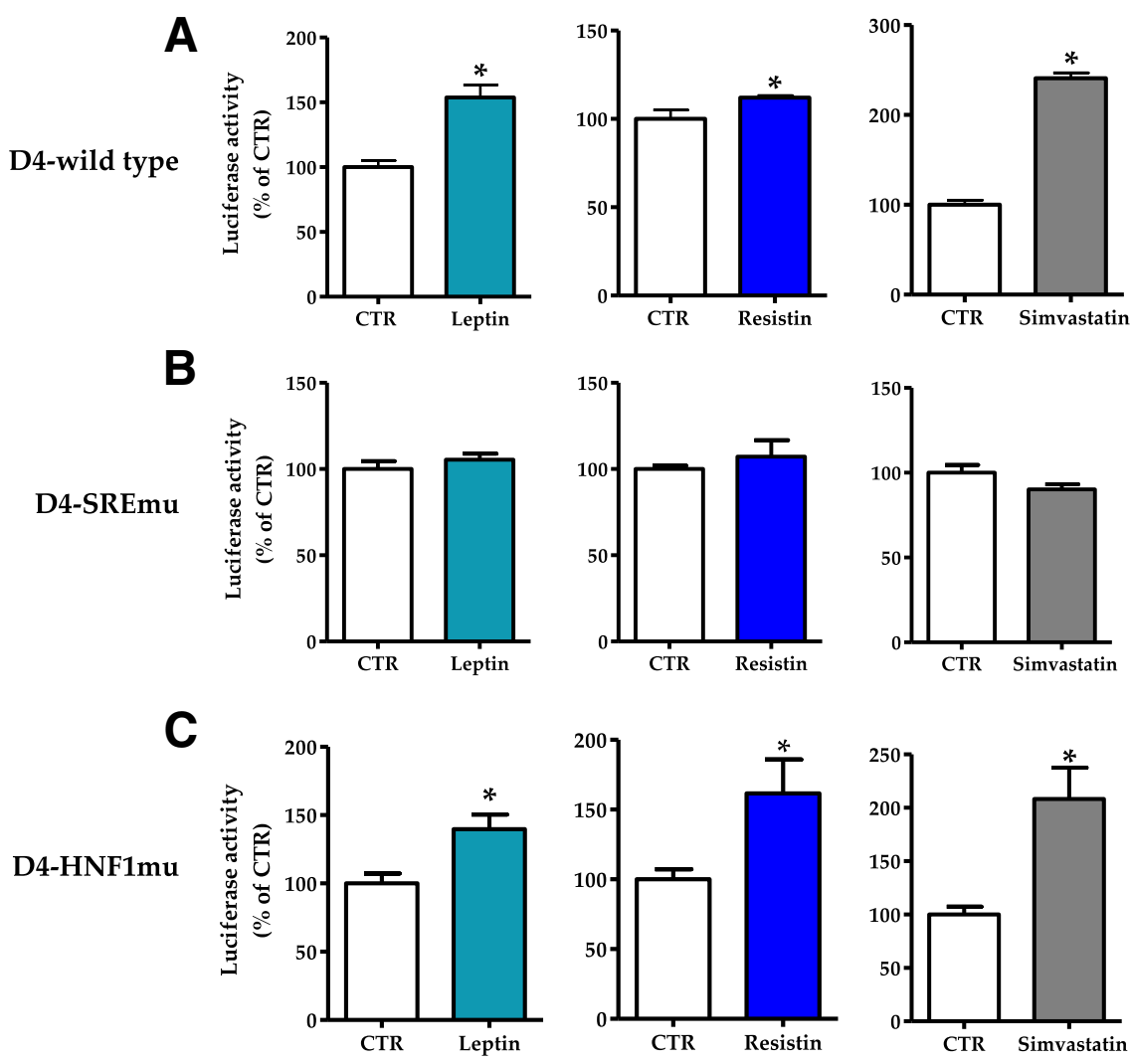

Figure 4 HepG2 cells were transfected with pGL3-PCSK9-D4 (A), pGL3-PCSK9-SREmut (B), and pGL3-PCSK9-HNF1mut (C). The day after the transfection, the medium was replaced with Dulbecco's modified Eagle's medium containing 10\% LPDS supplemented with leptin $(100 \mathrm{ng} / \mathrm{mL})$, resistin $(100 \mathrm{ng} / \mathrm{mL})$, or simvastatin $(20 \mu \mathrm{mol} / \mathrm{L})$. After an additional 24 hours, luciferase activities are determined by Neolite reagent. At least three independent experiments were conducted. ${ }^{*} P<0.05$ versus control ( $t$-test). CTR, control (medium alone). LPDS, human lipoprotein plasma deprived serum; HNF1, hepatocyte nuclear factor1; PCSK9, proprotein convertase subtilisin/kexin type 9; SRE, sterol regulatory element.
D1 and D1-STAT3-mut plasmids (Figure 3B), both containing the human PCSK9 promoter region spanning -1711 to -94 nucleotides upstream of the gene coding for the luciferase enzyme, HepG2 cells were treated with leptin, resistin, and simvastatin. A twenty-four-hour treatment enhanced transcriptional activity, an effect that was not counteracted when STAT3 mutation was inserted (Figure 3C). Simvastatin was used as a positive control (Figure 3D). These data suggest that PCSK 9 up-regulation by leptin and resistin in hepatocytes is not directly mediated by binding of STAT3 to the PCSK 9 promoter.

\section{Leptin and Resistin Affect the Transcriptional Activity of PCSK9}

To further explore the mechanism whereby leptin and resistin up-regulate PCSK9 transcript levels, HepG2 cells were transiently transfected with constructs containing the proximal human PCSK9 promoter region ( -440 to -94$)$. Leptin and resistin significantly incremented the luciferase activity by $54 \%$ and $15 \%$, respectively (Figure 4 A). Similar results were obtained upon treatment with simvastatin $(+141 \%)$. Because SREBPs and HNF-1 are the major transcription factors regulating PCSK $9,{ }^{26}$ luciferase activity was investigated upon the insertion of SRE and HNF-1 mutations in the PCSK 9 promoter sequence. The presence of mutation in the SRE sequence completely abolished the
PCSK9 transcriptional activity driven by leptin and resistin, as well as that of simvastatin (Figure 4B). Conversely, mutation in HNF-1 did not alter leptin- and resistin-driven luciferase activities, showing an increment of $43 \%$ and $58 \%$, respectively (Figure $4 \mathrm{C}$ ).

\section{Association Studies}

The circulating levels of leptin and PCSK9 were measured by ELISA in a clinical setting of 149 males ( $56 \pm 4$ years of age) free of CV disease at enrollment and belonging to the cohort of Brisighella Heart Study. ${ }^{22}$ A positive association between circulating leptin and PCSK9 levels ( $\beta=0.352$; $P=0.014)$ was found only in subjects with a body mass index $<25 \mathrm{~kg} / \mathrm{m}^{2}$ (Figure 5A). When the body mass index rose, that is, between 25 and $30 \mathrm{~kg} / \mathrm{m}^{2}(\beta=0.147$; $P=0.295)$ (Figure $5 \mathrm{~B})$ or $>30 \mathrm{~kg} / \mathrm{m}^{2}(\beta=-0.030$; $P=0.840$ ) (Figure $5 \mathrm{C}$ ), the association was lost. Concerning resistin, the circulating levels did not associate with PCSK9 (data not shown). The main characteristics of the selected participants have been reported in Supplemental Table S1.

\section{Discussion}

Obesity and associated metabolic disorders are becoming major health care concerns worldwide. Obesity is highly 


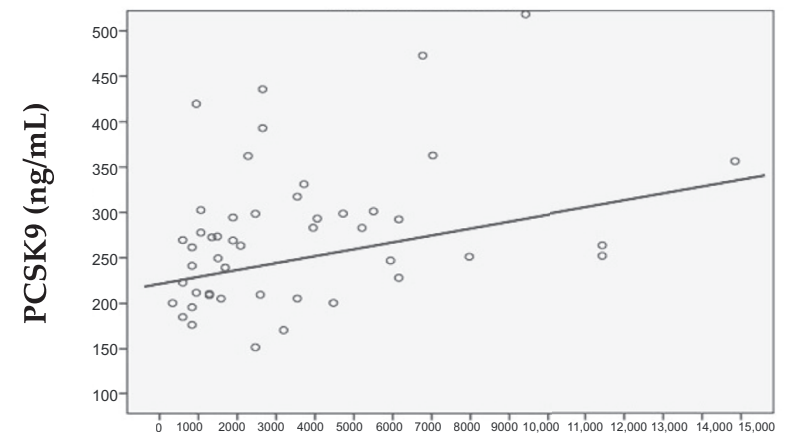

Leptin $(\mathrm{pg} / \mathrm{mL})$

B $25 \mathrm{~kg} / \mathrm{m}^{2}<\mathrm{BMI}<30 \mathrm{~kg} / \mathrm{m}^{2}$

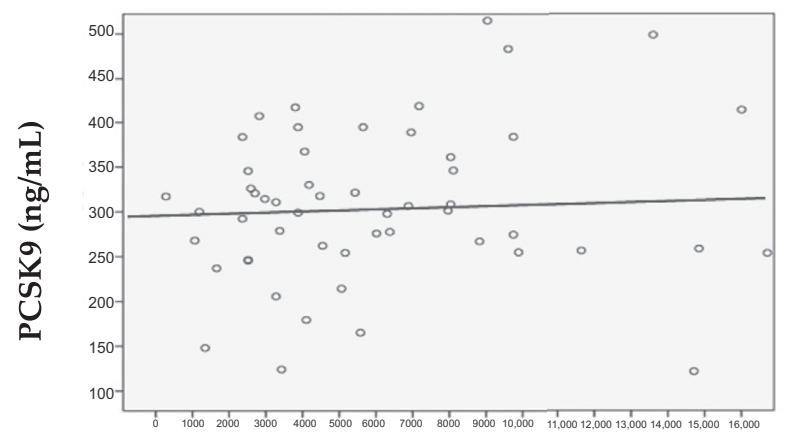

Leptin $(\mathrm{pg} / \mathrm{mL})$

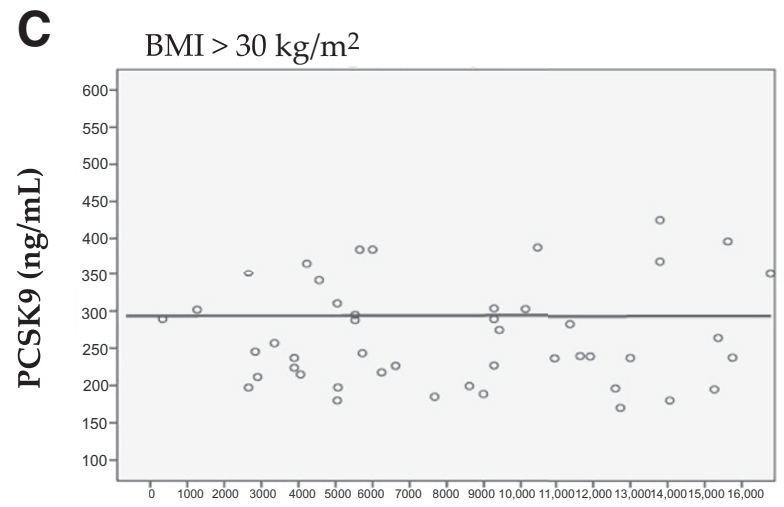

Leptin $(\mathrm{pg} / \mathrm{mL})$

Figure 5 Plasma levels of leptin and PCSK9 are measured by commercial ELISA. Subjects are stratified according to BMI, that is, $<25 \mathrm{~kg} / \mathrm{m}^{2}(\mathbf{A})$, between 25 and $30 \mathrm{~kg} / \mathrm{m}^{2}$ (B) and $>30 \mathrm{~kg} / \mathrm{m}^{2}$ (C). $n=48$ subjects (A and C); $n=53$ subjects (B). BMI, body mass index; ELISA, enzyme-linked immunosorbent assay; PCSK9, proprotein convertase subtilisin/kexin type 9.

associated with chronic low-grade inflammation, and it is believed that this obesity-linked inflammatory state is due to changes in the expression of cytokines released by adipose tissue. Adipose tissue is a source of secreted adipokines that act as modulators of metabolic and CV processes. ${ }^{27}$ Among adipokines, the expression of LDL receptors has been described to be modulated by leptin and resistin, the last being able to stabilize the cellular expression of PCSK9 ${ }^{5,28}$ Adiponectin has been also described to affect PCSK9 expression/production, although via the activation of PPAR $\gamma$ and AMPK pathways. ${ }^{29}$

In the tight liaison among adipokines, inflammation, and atherosclerosis, the main finding of this study relies on the evidence that leptin and resistin, two adipokines associated with regulation of atherogenesis and inflammation, induce the expression of PCSK9 in hepatocytes, which are the main source of circulating protein, through the Janus kinase (JAK)/STAT3 inflammatory pathway. A growing body of evidence suggests that this pathway is dysregulated in the context of obesity and metabolic disease. ${ }^{15}$ The interplay between inflammation and PCSK9 has been investigated in the last few years, leading to the hypothesis that it represents a fed-forward loop in which inflammation activates PCSK9, and vice versa, PCSK9 impacts the inflammatory burden associated with atheroma formation. ${ }^{30}$

The cytokine responsive JAK/STAT pathway is involved in adipokine-mediated crosstalk between adipocytes and liver or skeletal muscle. ${ }^{31,32}$ The involvement of STAT3 signaling upon leptin receptor activation represents the primary mechanism by which leptin regulates energy balance, ${ }^{33}$ resistin has also been reported to activate this pathway. ${ }^{34}$ In particular, STAT3, a transcription factor expressed in multiple metabolic tissues, is activated in response to cytokines, growth factors, and nutrients. ${ }^{35}$ STAT3 may account for the constitutive activation of NF- $\kappa \mathrm{B}$ during chronic inflammation. This activation contributes to the transcriptional regulation of inflammatory cytokines, such as ICAM-1, VCAM-1, MCP-1, and IL-6, as well as matrix metalloproteinases. ${ }^{36}$ Moreover, it has been shown that the impairment of oncostatin $\mathrm{M}$ signaling, a secreted cytokine mainly involved in chronic inflammatory and $\mathrm{CV}$ diseases, has a protective effect on atherosclerosis, partially due to the inhibition of the JAK2/STAT3 activation in macrophages. ${ }^{37}$ Thus, overall, by silencing STAT3, it has been demonstrated that this inflammatory pathway may be at the crossroad between adipose tissue and PCSK9 activation. Furthermore, STAT3 phosphorylation is markedly increased in atherosclerotic lesions, and interfering with STAT3 pathway prevents atherosclerotic lesion formation in vivo. ${ }^{38}$ In line with this evidence, the direct role of PCSK9 in atheroma formation should be considered. The Atherosclerosis Risk in Communities (ARIC) epidemiologic study demonstrated that genetically reduced levels of PCSK9 were associated with a reduction in myocardial infarction, fatal coronary heart diseases, or coronary revascularization, despite a significant prevalence of other nonlipid-related CV risk factors. ${ }^{39}$ Higher serum PCSK9 levels were linearly associated with a higher necrotic core fraction in coronary atherosclerosis, regardless of serum LDL-cholesterol, confirming data in PCSK9 knockout mice that were partially protected from neointimal formation. ${ }^{40}$ In humans, PCSK9 has been also associated with other components of atheroma formation, that is, platelet aggregation, ${ }^{16}$ hypertension, and inflammation. ${ }^{30}$

It has been found that leptin and resistin activate the transcription of PCSK9 through the involvement of the SRE 
motif, apparently without the involvement of HNF- $1 .^{41}$ The involvement of the STAT3 pathway at the transcriptional level was negligible. However, this latter analysis requires further investigation because the promoter region of human PCSK9 contains other STAT3 responsible elements (Table 2). Because PCSK9 transcription is controlled through cis-regulatory elements imbedded in the proximal promoter region of PCSK9 gene where the Sp1 sites, SRE and HNF-1, are located, a PCSK9 promoter luciferase reporter plasmid containing mutations for both SRE and HNF responsive elements was used. It was found that exposure of HepG2 hepatocytes to leptin and resistin increases the transcriptional activity of the PCSK 9 promoter. Furthermore, although a construct variant containing a mutation in the HNF-1 motif did not affect the ability of adipokines to up-regulate luciferase activity, no induction was detected after introducing a mutation in the SRE motif. Relative to this latter evidence, the current study data are in line with findings demonstrating that, at the transcriptional level, PCSK9 synthesis is largely controlled by SREBPs. ${ }^{42}$ These findings are in agreement with the observation that leptin induces SREBP1 activation in porcine granulosa cells. ${ }^{43}$

When looking at the correlation between circulating levels of PCSK9 and leptin, it was found that this exists only in the group with a body mass index $<25 \mathrm{~kg} / \mathrm{m}^{2}$, an evidence in line with that previous described by Kwakernaak et $\mathrm{al}^{44}$ in 30 women. This becomes relevant considering that both PCSK9 and leptin levels follow a gender difference, being higher in female subjects than in male. ${ }^{45-47}$ Overall, leptin may be a determinant of PCSK9 levels predominantly before a state of leptin resistance occurs, and most of all, in obese patients, other factors may impact on the regulation of PCSK9 levels. Leptin resistance, proposed to take place during the onset and progression of obesity, ${ }^{48}$ could be the result of an impaired leptin signaling pathway, including reduced access of the hormone to its receptor or due to epigenetic modifications that could contribute to leptin expression and signaling disturbances. ${ }^{49}$ This complex scenario has been also highlighted in ob/ob mice lacking leptin in which the effects of leptin replacement on PCSK9 mRNA expression and protein levels were sex-dependent. ${ }^{50}$ In male mice, leptin treatment reduced plasma levels of PCSK9, whereas no changes were observed in females. ${ }^{50}$ On the other hand, although it was suggested that resistin may be involved in the interplay between inflammation and atherosclerosis, whether circulating resistin is a determinant of higher PCSK9 levels needs to be further explored. ${ }^{44}$ Indeed, this study's data obtained in healthy male subjects did not fit with a previous report by $\mathrm{Li}$ et al, ${ }^{51}$ who found that in patients with $\mathrm{CV}$ artery disease resistin positively correlate with PCSK9 levels. Some of these discrepancies may also rely on the tight liaison surrounding resistin and PCSK9, that is, the C-terminal domain of PCSK9 has a structural homology with the human resistin C-terminal domain, and CAP1 represents a binding partner of PCSK9 in mediating LDL receptor degradation. ${ }^{52}$ On this matter, the authors have previously demonstrated that PCSK9 induces a proinflammatory response in macrophages,${ }^{53}$ an effect common to resistin, that is, a rise in IL6, TNF- $\alpha$, and MCP-1 (data not published). Conversely, a possible vicious circle in the liver has been excluded based on the observation that treatment with human recombinant PCSK9 did not increase the gene expression of IL6 in HepG2 cells (Supplemental Figure S3). This is in line with recent findings showing that PCSK9 is not induced in artificial human inflammation and is not correlated with inflammatory response. ${ }^{54}$

Overall, the study provides evidence that PCSK9 may be one of the players at the crossroad of the interplay between obesity, adipose tissue dysfunction, and $\mathrm{CV}$ disease risk. Such effect appears to be dependent on the inflammatory JAK/STAT pathway.

\section{Supplemental Data}

Supplemental material for this article can be found at http://doi.org/10.1016/j.ajpath.2020.07.016.

\section{References}

1. Perez-Tilve D: 'Et tu, leptin?'. Trends Endocrinol Metab 2019, 30: 232-233

2. Barale C, Russo I: Influence of cardiometabolic risk factors on platelet function. Int J Mol Sci 2020, 21:623

3. Ades PA, Savage PD: Obesity in coronary heart disease: an unaddressed behavioral risk factor. Prev Med 2017, 104:117-119

4. Rashid S, Kastelein JJ: PCSK9 and resistin at the crossroads of the atherogenic dyslipidemia. Expert Rev Cardiovasc Ther 2013, 11: $1567-1577$

5. Du Y, Li S, Cui C-J, Zhang Y, Yang S-H, Li J-J: Leptin decreases the expression of low-density lipoprotein receptor via PCSK9 pathway: linking dyslipidemia with obesity. J Transl Med 2016, 14:276

6. Cui H, Lopez M, Rahmouni K: The cellular and molecular bases of leptin and ghrelin resistance in obesity. Nat Rev Endocrinol 2017, 13 338-351

7. Banks AS, Davis SM, Bates SH, Myers MG Jr: Activation of downstream signals by the long form of the leptin receptor. J Biol Chem 2000, 275:14563-14572

8. Gao Q, Wolfgang MJ, Neschen S, Morino K, Horvath TL, Shulman GI, Fu X-Y: Disruption of neural signal transducer and activator of transcription 3 causes obesity, diabetes, infertility, and thermal dysregulation. Proc Natl Acad Sci U S A 2004, 101: 4661-4666

9. Bates SH, Stearns WH, Dundon TA, Schubert M, Tso AW, Wang Y Banks AS, Lavery HJ, Haq AK, Maratos-Flier E, Neel BG, Schwartz MW, Myers MG Jr: STAT3 signalling is required for leptin regulation of energy balance but not reproduction. Nature 2003, 421: 856-859

10. Reilly MP, Lehrke M, Wolfe ML, Rohatgi A, Lazar MA, Rader DJ Resistin is an inflammatory marker of atherosclerosis in humans Circulation 2005, 111:932-939

11. Lee S, Lee H-C, Kwon Y-W, Lee SE, Cho Y, Kim J, Lee S, Kim J-Y, Lee J, Yang H-M, Mook-Jung I, Nam K-Y, Chung J, Lazar MA, Kim H-S: Adenylyl cyclase-associated protein 1 is a receptor for human resistin and mediates inflammatory actions of human monocytes. Cell Metab 2014, 19:484-497

12. Macchi C, Banach M, Corsini A, Sirtori CR, Ferri N, Ruscica M: Changes in circulating pro-protein convertase subtilisin/kexin type 9 levels - experimental and clinical approaches with lipid-lowering agents. Eur J Prev Cardiol 2019, 26:930-949 
13. Momtazi-Borojeni AA, Sabouri-Rad S, Gotto AM, Pirro M, Banach M, Awan Z, Barreto GE, Sahebkar A: PCSK9 and inflammation: a review of experimental and clinical evidence. Eur Heart $\mathrm{J}$ Cardiovasc Pharmacother 2019, 5:237-245

14. Ruscica M, Baragetti A, Catapano AL, Norata GD: Translating the biology of adipokines in atherosclerosis and cardiovascular diseases: gaps and open questions. Nutr Metab Cardiovasc Dis 2017, 27: 379-395

15. Dodington DW, Desai HR, Woo M: JAK/STAT - emerging players in metabolism. Trends Endocrinol Metab 2018, 29:55-65

16. Camera M, Rossetti L, Barbieri SS, Zanotti I, Canciani B, Trabattoni D, Ruscica M, Tremoli E, Ferri N: PCSK9 as a positive modulator of platelet activation. J Am Coll Cardiol 2018, 71: 952-954

17. Yates AD, Achuthan P, Akanni W, Allen J, Allen J, Alvarez-Jarreta J, et al: Ensembl 2020. Nucleic Acids Res 2020, 48:D682-D688

18. Fornes O, Castro-Mondragon JA, Khan A, van der Lee R, Zhang X, Richmond PA, Modi BP, Correard S, Gheorghe M, Baranasic D, Santana-Garcia W, Tan G, Cheneby J, Ballester B, Parcy F, Sandelin A, Lenhard B, Wasserman WW, Mathelier A: JASPAR 2020: update of the open-access database of transcription factor binding profiles. Nucleic Acids Res 2020, 48:D87-D92

19. Grant CE, Bailey TL, Noble WS: FIMO: scanning for occurrences of a given motif. Bioinformatics 2011, 27:1017-1018

20. Bailey TL, Boden M, Buske FA, Frith M, Grant CE, Clementi L, Ren J, Li WW, Noble WS: MEME SUITE: tools for motif discovery and searching. Nucleic Acids Res 2009, 37:W202-W208

21. Li H, Dong B, Park SW, Lee H-S, Chen W, Liu J: Hepatocyte nuclear factor 1alpha plays a critical role in PCSK9 gene transcription and regulation by the natural hypocholesterolemic compound berberine. $\mathrm{J}$ Biol Chem 2009, 284:28885-28895

22. Ruscica M, Ferri N, Fogacci F, Rosticci M, Botta M, Marchiano S, Magni P, D'Addato S, Giovannini M, Borghi C, Cicero AFG; Brisighella Heart Study Group: Circulating levels of proprotein convertase subtilisin/kexin type 9 and arterial stiffness in a large population sample: data from the Brisighella Heart Study. J Am Heart Assoc 2017, 6:e005764

23. Ruscica M, Simonelli S, Botta M, Ossoli A, Lupo MG, Magni P, Corsini A, Arca M, Pisciotta L, Veglia F, Franceschini G, Ferri N, Calabresi L: Plasma PCSK9 levels and lipoprotein distribution are preserved in carriers of genetic HDL disorders. Biochim Biophys Acta Mol Cell Biol Lipids 2018, 1863:991-997

24. Ruscica M, Macchi C, Gandini S, Morlotti B, Erzegovesi S, Bellodi L, Magni P: Free and bound plasma leptin in anorexia nervosa patients during a refeeding program. Endocrine 2016, 51: 380-383

25. Tripathi SK, Chen Z, Larjo A, Kanduri K, Nousiainen K, Aijo T, Ricano-Ponce I, Hrdlickova B, Tuomela S, Laajala E, Salo V, Kumar V, Wijmenga C, Lahdesmaki H, Lahesmaa R: Genome-wide analysis of STAT3-mediated transcription during early human Th17 cell differentiation. Cell Rep 2017, 19:1888-1901

26. Ruscica M, Ricci C, Macchi C, Magni P, Cristofani R, Liu J, Corsini A, Ferri N: Suppressor of cytokine signaling-3 (SOCS-3) induces proprotein convertase subtilisin kexin type 9 (PCSK9) expression in hepatic HepG2 cell line. J Biol Chem 2016, 291: 3508-3519

27. Neeland IJ, Ross R, Despres JP, Matsuzawa Y, Yamashita S, Shai I, Seidell J, Magni P, Santos RD, Arsenault B, Cuevas A, Hu FB, Griffin B, Zambon A, Barter P, Fruchart JC, Eckel RH; International Atherosclerosis Society; International Chair on Cardiometabolic Risk Working Group on Visceral Obesity: Visceral and ectopic fat, atherosclerosis, and cardiometabolic disease: a position statement. Lancet Diabetes Endocrinol 2019, 7:715-725

28. Melone M, Wilsie L, Palyha O, Strack A, Rashid S: Discovery of a new role of human resistin in hepatocyte low-density lipoprotein receptor suppression mediated in part by proprotein convertase subtilisin/kexin type 9. J Am Coll Cardiol 2012, 59:1697-1705
29. Sun L, Yang X, Li Q, Zeng P, Liu Y, Liu L, Chen Y, Yu M, Ma C, Li X, Li Y, Zhang R, Zhu Y, Miao QR, Han J, Duan Y: Activation of adiponectin receptor regulates proprotein convertase subtilisin/kexin type 9 expression and inhibits lesions in ApoE-deficient mice. Arterioscler Thromb Vasc Biol 2017, 37:1290-1300

30. Ruscica M, Tokgozoglu L, Corsini A, Sirtori CR: PCSK9 inhibition and inflammation: a narrative review. Atherosclerosis 2019, 288:146-155

31. Emanuelli B, Peraldi P, Filloux C, Chavey C, Freidinger K, Hilton DJ, Hotamisligil GS, Van Obberghen E: SOCS-3 inhibits insulin signaling and is up-regulated in response to tumor necrosis factor-alpha in the adipose tissue of obese mice. J Biol Chem 2001, 276:47944-47949

32. Ueki K, Kondo T, Kahn CR: Suppressor of cytokine signaling 1 (SOCS-1) and SOCS-3 cause insulin resistance through inhibition of tyrosine phosphorylation of insulin receptor substrate proteins by discrete mechanisms. Mol Cell Biol 2004, 24:5434-5446

33. Allison MB, Myers MG Jr: 20 years of leptin: connecting leptin signaling to biological function. J Endocrinol 2014, 223:T25-T35

34. Pirvulescu M, Manduteanu I, Gan AM, Stan D, Simion V, Butoi E, Calin M, Simionescu M: A novel pro-inflammatory mechanism of action of resistin in human endothelial cells: up-regulation of SOCS3 expression through STAT3 activation. Biochem Biophys Res Commun 2012, 422:321-326

35. Mashili F, Chibalin AV, Krook A, Zierath JR: Constitutive STAT3 phosphorylation contributes to skeletal muscle insulin resistance in type 2 diabetes. Diabetes 2013, 62:457-465

36. Bode JG, Albrecht U, Haussinger D, Heinrich PC, Schaper F: Hepatic acute phase proteins-regulation by IL-6- and IL-1-type cytokines involving STAT3 and its crosstalk with NF-kappaB-dependent signaling. Eur J Cell Biol 2012, 91:496-505

37. Zhang X, Li J, Qin J-J, Cheng W-L, Zhu X, Gong F-H, She Z, Huang Z, Xia H, Li H: Oncostatin M receptor beta deficiency attenuates atherogenesis by inhibiting JAK2/STAT3 signaling in macrophages. J Lipid Res 2017, 58:895-906

38. Dutzmann J, Daniel JM, Bauersachs J, Hilfiker-Kleiner D, Sedding DG: Emerging translational approaches to target STAT3 signalling and its impact on vascular disease. Cardiovasc Res 2015, 106:365-374

39. Cohen JC, Boerwinkle E, Mosley TH Jr, Hobbs HH: Sequence variations in PCSK9, low LDL, and protection against coronary heart disease. N Engl J Med 2006, 354:1264-1272

40. Ferri N, Marchiano S, Tibolla G, Baetta R, Dhyani A, Ruscica M, Uboldi P, Catapano AL, Corsini A: PCSK9 knock-out mice are protected from neointimal formation in response to perivascular carotid collar placement. Atherosclerosis 2016, 253:214-224

41. Dong B, Singh AB, Shende VR, Liu J: Hepatic HNF1 transcription factors control the induction of PCSK9 mediated by rosuvastatin in normolipidemic hamsters. Int J Mol Med 2017, 39:749-756

42. Jeong HJ, Lee H-S, Kim K-S, Kim Y-K, Yoon D, Park SW: Steroldependent regulation of proprotein convertase subtilisin/kexin type 9 expression by sterol-regulatory element binding protein-2. J Lipid Res 2008, 49:399-409

43. Ruiz-Cortes ZT, Martel-Kennes Y, Gevry NY, Downey BR, Palin MF, Murphy BD: Biphasic effects of leptin in porcine granulosa cells. Biol Reprod 2003, 68:789-796

44. Kwakernaak AJ, Lambert G, Dullaart RP: Plasma proprotein convertase subtilisin-kexin type 9 is predominantly related to intermediate density lipoproteins. Clin Biochem 2014, 47:679-682

45. Lakoski SG, Lagace TA, Cohen JC, Horton JD, Hobbs HH: Genetic and metabolic determinants of plasma PCSK9 levels. J Clin Endocrinol Metab 2009, 94:2537-2543

46. Ooi TC, Raymond A, Cousins M, Favreau C, Taljaard M, Gavin C, Jolly EE, Malone S, Eapen L, Chretien M, Mbikay M, Mayne J: Relationship between testosterone, estradiol and circulating PCSK9: cross-sectional and interventional studies in humans. Clin Chim Acta 2015, 446:97-104

47. Macchi C, Ferri N, Favero C, Cantone L, Vigna L, Pesatori AC, Lupo MG, Sirtori CR, Corsini A, Bollati V, Ruscica M: Long-term 
exposure to air pollution raises circulating levels of proprotein convertase subtilisin/kexin type 9 in obese individuals. Eur J Prev Cardiol 2019, 26:578-588

48. Munzberg H, Myers MG Jr: Molecular and anatomical determinants of central leptin resistance. Nat Neurosci 2005, 8:566-570

49. Crujeiras AB, Carreira MC, Cabia B, Andrade S, Amil M, Casanueva FF: Leptin resistance in obesity: an epigenetic landscape. Life Sci 2015, 140:57-63

50. Levenson AE, Haas ME, Miao J, Brown RJ, de Ferranti SD, Muniyappa R, Biddinger SB: Effect of leptin replacement on PCSK9 in ob/ob mice and female lipodystrophic patients. Endocrinology 2016, 157:1421-1429

51. Li S, Xu RX, Zhang Y, Guo YL, Zhu CG, Liu G, Dong Q, Li JJ: Relation of resistin to proprotein convertase subtilisin-kexin type 9 levels in coronary artery disease patients with different nutritional status. J Endocrinol Invest 2015, 38:1291-1299
52. Jang H-D, Lee SE, Yang J, Lee H-C, Shin D, Lee H, Lee J, Jin S, Kim S, Lee SJ, You J, Park H-W, Nam K-Y, Lee S-H, Park SW, Kim J-S, Kim S-Y, Kwon Y-W, Kwak SH, Yang H-M, Kim H-S: Cyclase-associated protein 1 is a binding partner of proprotein convertase subtilisin/kexin type- 9 and is required for the degradation of low-density lipoprotein receptors by proprotein convertase subtilisin/kexin type-9. Eur Heart J 2020, 41:239-252

53. Ricci C, Ruscica M, Camera M, Rossetti L, Macchi C, Colciago A, Zanotti I, Lupo MG, Adorni MP, Cicero AFG, Fogacci F, Corsini A, Ferri N: PCSK9 induces a pro-inflammatory response in macrophages. Sci Rep 2018, 8:2267

54. Heinzl MW, Resl M, Klammer C, Egger M, Dieplinger B, Clodi M: Proprotein convertase subtilisin/kexin type 9 (PCSK9) is not induced in artificial human inflammation and is not correlated with inflammatory response. Infect Immun 2020, 88:e0842-19 